\title{
Fermi-Surface Curvature and Hall Conductivity in Metals
}

\author{
Osamu Narikiyo*
}

2020-11-02

\begin{abstract}
The Tsuji formula which relates the Fermi-surface curvature and the weak-field Hall conductivity in metals is discussed in Haldane's framework.
\end{abstract}

\section{Introduction}

The Tsuji formula [1] relates the Fermi-surface curvature and the weak-field Hall conductivity in metals. Although it is widely known as a geometrical formula, its intelligible derivation has not been reported. For example, in the Appendix of an influential textbook [2] the author reported the correspondence with Tsuji but it does not seem a derivation. I think that not only the derivation but also the meaning of the Tsuji formula becomes clear when we employ Haldane's framework. [3] Thus we will discuss the Tsuji formula in Haldane's framework.

While the Tsuji formula [1] was derived under the assumption of the cubic symmetry, Haldane [3] tried to eliminate the assumption. However, we will criticize the trial.

This paper is organized as follows. In the section 2 we start from the Boltzmann transport theory. In the section 3 we focus on the contribution from the Fermi surface. We review and criticize Haldane's result in the section 4. In the section 5 we analyze the Tsuji formula in Haldane's framework. Another geometrical formula by Ong [4] is briefly mentioned in the section 6 . We give short summary in the last section.

\section{Boltzmann conductivity}

The weak-field DC Hall conductivity $\sigma^{x y}$ per spin is given by

$$
\sigma^{x y}=e^{3} \sum_{\boldsymbol{k}}\left(-\frac{\partial f}{\partial \varepsilon}\right) l^{x}(\boldsymbol{B} \times \boldsymbol{v}) \cdot \frac{\partial}{\partial \boldsymbol{k}} l^{y},
$$

using the solution of the linearized Boltzmann equation. [2, 5] Here the magnetic field $\boldsymbol{B}$ is a constant vector. The mean free path vector $\boldsymbol{l}=\left(l^{x}, l^{y}, l^{z}\right)$ is given as $\boldsymbol{l}=\boldsymbol{\tau} \boldsymbol{v}$

*Department of Physics, Kyushu University 
where $\boldsymbol{v}=\left(v^{x}, v^{y}, v^{z}\right)$ is the velocity of the quasi-particle and $\tau$ is its renormalized transport life-time. $\partial f / \partial \varepsilon$ is the derivative of the Fermi distribution function $f$ with respect to the quasi-particle energy $\varepsilon$. Although we have suppressed the argument of $\boldsymbol{v}, \tau$ and $\varepsilon$, they are the functions of the position $\boldsymbol{k}=\left(k^{x}, k^{y}, k^{z}\right)$ in $\boldsymbol{k}$-space. The component of the quasi-particle velocity $v^{a}(a=x, y, z)$ is given as $v^{a}=\partial \varepsilon / \partial k^{a} \equiv \varepsilon_{a}$.

It should be noticed that Eq. (1) is not the consequence of the relaxation-time approximation but the effects of the collision term is fully taken into account by renormalizing the life-time. [6] Thus Eq. (1) is in accordance with the result of the Fermi-liquid theory. [7]

The off-diagonal conductivity, Eq. (1), satisfies the Onsager's reciprocal relation: $\sigma_{x y}=-\sigma_{y x}$ with

$$
\sigma^{y x}=e^{3} \sum_{\boldsymbol{k}}\left(-\frac{\partial f}{\partial \varepsilon}\right) l^{y}(\boldsymbol{B} \times \boldsymbol{v}) \cdot \frac{\partial}{\partial \boldsymbol{k}} l^{x} .
$$

Actually the integration by parts [5] for $\sigma_{x y}$ leads to $-\sigma_{y x}$, since

$$
\frac{\partial}{\partial \boldsymbol{k}} \frac{\partial f}{\partial \varepsilon} \cdot(\boldsymbol{B} \times \boldsymbol{v})=0,
$$

and

$$
\frac{\partial}{\partial \boldsymbol{k}} \cdot(\boldsymbol{B} \times \boldsymbol{v})=0 .
$$

Eq. (3) and Eq. (4) are the consequences of $v=\partial \varepsilon / \partial \boldsymbol{k}$.

In the following we switch the factor $(\boldsymbol{B} \times \boldsymbol{v}) \cdot \partial / \partial \boldsymbol{k}$ to its equivalence $(\boldsymbol{v} \times \partial / \partial \boldsymbol{k}) \cdot \boldsymbol{B}$. Then Eq. (1) is equivalent to

$$
\sigma^{x y}=e^{3} \sum_{\boldsymbol{k}}\left(-\frac{\partial f}{\partial \varepsilon}\right) l^{x}\left(\boldsymbol{v} \times \frac{\partial}{\partial \boldsymbol{k}}\right) \cdot \boldsymbol{B} l^{y} .
$$

If we measure the Hall current in $x$-direction, $J^{x}$, under the electric field in $y$ direction, $\boldsymbol{E}=\left(0, E^{y}, 0\right)$, and the magnetic field in $z$-direction, $\boldsymbol{B}=\left(0,0, B^{z}\right), J^{x}=$ $\sigma^{x z y} B^{z} E^{y}$ with

$$
\sigma^{x z y}=e^{3} \sum_{\boldsymbol{k}}\left(-\frac{\partial f}{\partial \varepsilon}\right) l^{x}\left(\boldsymbol{v} \times \frac{\partial}{\partial \boldsymbol{k}}\right)^{z} l^{y} .
$$

Since $\sigma^{x z y}=-\sigma^{y z x}$, we use $e^{3} \gamma^{x z y} \equiv\left(\sigma_{x z y}-\sigma_{y z x}\right) / 2$ instead of $\sigma^{x z y}$. The antisymmetric property, $\gamma^{x z y}=-\gamma^{y z x}$, is evident in

$$
\gamma^{x z y}=\frac{1}{2} \sum_{\boldsymbol{k}}\left(-\frac{\partial f}{\partial \varepsilon}\right)\left[l^{x}\left(\boldsymbol{v} \times \frac{\partial}{\partial \boldsymbol{k}}\right)^{z} l^{y}-l^{y}\left(\boldsymbol{v} \times \frac{\partial}{\partial \boldsymbol{k}}\right)^{z} l^{x}\right] .
$$

Using

$$
\sigma^{x z y}=e^{3} \sum_{\boldsymbol{k}}\left(-\frac{\partial f}{\partial \varepsilon}\right) \tau v^{x}\left(v^{x} \frac{\partial}{\partial k^{y}}-v^{y} \frac{\partial}{\partial k^{x}}\right) \tau v^{y}
$$

we obtain

$$
\gamma^{x z y}=\frac{1}{2} \sum_{\boldsymbol{k}}\left(-\frac{\partial f}{\partial \varepsilon}\right)\left(v^{x}, v^{y}\right)\left(\begin{array}{cc}
M_{y y}^{-1} & -M_{y x}^{-1} \\
-M_{x y}^{-1} & M_{x x}^{-1}
\end{array}\right)\left(\begin{array}{l}
v^{x} \\
v^{y}
\end{array}\right) \tau^{2}
$$


where

$$
M_{a b}^{-1} \equiv \frac{\partial^{2} \varepsilon}{\partial k^{a} \partial k^{b}} \equiv \varepsilon_{a b},
$$

is the effective mass tensor. Through the subtraction, $\sigma^{x y z}-\sigma^{y x z}$, the derivatives of $\tau$ cancel out. This result (9) is equivalent to that of the diagrammatic analysis [7] of the linear response of the Fermi liquid. Thus the expression for the Hall conductivity which contains the derivatives of $\tau$, for example (2.53) in Ref. [2], is a bad expression.

Eq. (9) is the general result for the Hall conductivity so that you have only to estimate it numerically if you are not interested in its geometrical interpretation.

\section{Fermi-surface contribution}

In the case of Fermi degeneracy we can estimate $-\partial f / \partial \varepsilon$ by the delta function:

$$
\int \mathrm{d} \boldsymbol{k}\left(-\frac{\partial f}{\partial \varepsilon}\right)=\int \frac{\mathrm{d} S}{|\boldsymbol{v}|},
$$

where $\boldsymbol{v}=\partial \varepsilon / \partial \boldsymbol{k}$ and the integral in the right-hand-side is over the Fermi surface. Thus $\gamma^{x z y}$ is determined by the integration over the Fermi surface:

$$
\gamma^{x z y}=\frac{1}{2} \int \frac{\mathrm{d} S}{(2 \pi)^{3}}\left(v^{x}, v^{y}\right)\left(\begin{array}{cc}
M_{y y}^{-1} & -M_{y x}^{-1} \\
-M_{x y}^{-1} & M_{x x}^{-1}
\end{array}\right)\left(\begin{array}{l}
v^{x} \\
v^{y}
\end{array}\right) \frac{\tau^{2}}{|v|},
$$

and reflects the geometry of the Fermi surface. Actually it is shown in the following that $\gamma^{x z y}$ is a sampling of the local curvatures of the Fermi surface.

Throughout this paper we only consider the contribution from a single sheet of the Fermi surface. In the case of multi-sheets we should sum the contributions from all the sheets. [3]

\section{Haldane's framework}

Haldane used the framework

$$
\sigma^{x y} \equiv e^{3} \sum_{a} \sum_{b} \epsilon^{x y a} \gamma_{a b} B^{b}
$$

where $\epsilon^{a b c}$ is the antisymmetric rank-3 Levi-Civita symbol. Since Haldane derived $\gamma_{a b}$ from (1), the last equation in p. 2 of Ref. $3, \epsilon^{x y z} \gamma_{z z}$ should be equal to our $\gamma^{x z y}$. However, it is different from ours:

$$
\gamma_{z z}=\frac{1}{2} \int \frac{\mathrm{d} S}{(2 \pi)^{3}}\left(n^{x}, n^{y}\right)\left(\begin{array}{cc}
\kappa^{y y} & -\kappa^{y x} \\
-\kappa^{x y} & \kappa^{x x}
\end{array}\right)\left(\begin{array}{l}
n^{x} \\
n^{y}
\end{array}\right) l^{2},
$$

where $\boldsymbol{n}$ is the unit vector, $\boldsymbol{n} \equiv \boldsymbol{v} /|\boldsymbol{v}| \equiv\left(n^{x}, n^{y}, n^{z}\right)$, which is normal to the Fermi surface, $\kappa^{a b} \equiv \partial n^{a} / \partial k^{b}$ and $l^{2}=|\boldsymbol{l}|^{2}=|\boldsymbol{v}|^{2} \tau^{2}$. For example, ours needs only $\left(\partial v^{x} / \partial k^{y}\right) /|v|$ but Haldane's needs $\partial\left(v^{x} /|v|\right) / \partial k^{y}$. As discussed in the Appendices 
$\kappa^{a b}$ is a key ingredient of the geometrical description but its appearance in (14) is incorrect. Since it has no geometrical meaning, the life-time $\tau$ is safely separated from the other factors related to the shape of the Fermi surface as (12). On the other hand, in Eq. (14) the mean free path $l$ which still contains the geometrical information of the Fermi surface is separated. Such a separation is a bad strategy and cannot be justified. Anyway Eq. (14) is not derived from the solution of the Boltzmann equation (1).

The information on the geometry of the Fermi surface can be obtained from the $3 \times 3$ matrix

$$
\left(\begin{array}{lll}
\kappa^{x x} & \kappa^{y x} & \kappa^{z x} \\
\kappa^{x y} & \kappa^{y y} & \kappa^{z y} \\
\kappa^{x z} & \kappa^{y z} & \kappa^{z z}
\end{array}\right)
$$

It can be diagonalized as

$$
\left(\begin{array}{ccc}
\kappa_{1} & 0 & 0 \\
0 & \kappa_{2} & 0 \\
0 & 0 & 0
\end{array}\right)
$$

The pair of the eigenvalues $\left(\kappa_{1}\right.$ and $\left.\kappa_{2}\right)$ is the basis of the geometrical interpretation: $G=\kappa_{1} \kappa_{2}$ and $2 H=\kappa_{1}+\kappa_{2}$ where $G$ is the Gaussian curvature and $H$ is the mean curvature.

Especially the trace $2 H$, which is independent of the choice of the local coordinate, is the target in the next section.

\section{Tsuji formula in 3D}

Our master equation (12) is rewritten as

$$
\gamma^{x z y}=\int \frac{\mathrm{d} S}{(2 \pi)^{3}} h_{z} \tau^{2}
$$

where $h_{z}$ is determined by the derivative of the function $\varepsilon$ which determines the shape of the Fermi surface:

$$
h_{z}=\frac{1}{2|v|}\left(\varepsilon_{x} \varepsilon_{x} \varepsilon_{y y}+\varepsilon_{y} \varepsilon_{y} \varepsilon_{x x}-\varepsilon_{x} \varepsilon_{y} \varepsilon_{y x}-\varepsilon_{y} \varepsilon_{x} \varepsilon_{x y}\right),
$$

with $\varepsilon_{a}=\partial \varepsilon / \partial k^{a}$ and $\varepsilon_{a b}=\partial^{2} \varepsilon / \partial k^{a} \partial k^{b}$. This $h_{z}$ reflects the Fermi-surface geometry but $\tau$ has no geometrical meaning.

On the other hand, the trace $2 H=\kappa^{x x}+\kappa^{y y}+\kappa^{z z}$ of the $3 \times 3$ representation (15) becomes

$$
\begin{aligned}
& 2 H=\frac{1}{|\boldsymbol{v}|^{3}}\left[\varepsilon_{x} \varepsilon_{x}\left(\varepsilon_{y y}+\varepsilon_{z z}\right)+\varepsilon_{y} \varepsilon_{y}\left(\varepsilon_{z z}+\varepsilon_{x x}\right)\right. \\
& +\varepsilon_{z} \varepsilon_{z}\left(\varepsilon_{x x}+\varepsilon_{y y}\right)-\varepsilon_{x}\left(\varepsilon_{y} \varepsilon_{y x}+\varepsilon_{z} \varepsilon_{z x}\right) \\
& \left.-\varepsilon_{y}\left(\varepsilon_{x} \varepsilon_{x y}+\varepsilon_{z} \varepsilon_{z y}\right)-\varepsilon_{z}\left(\varepsilon_{x} \varepsilon_{x z}+\varepsilon_{y} \varepsilon_{y z}\right)\right] \text {, }
\end{aligned}
$$

as shown in the Appendix. The mean curvature $H$ is given by this expression (19) for any shape of the Fermi surface. 
By comparing (18) and (19) we see that $h_{z}$ is a piece of $H$. By summing three pieces we can construct $H: H=\left(h_{z}+h_{x}+h_{y}\right) /|v|^{2}$ where

$$
h_{x}=\frac{1}{2|v|}\left(\varepsilon_{y} \varepsilon_{y} \varepsilon_{z z}+\varepsilon_{z} \varepsilon_{z} \varepsilon_{y y}-\varepsilon_{y} \varepsilon_{z} \varepsilon_{z y}-\varepsilon_{z} \varepsilon_{y} \varepsilon_{y z}\right)
$$

and

$$
h_{y}=\frac{1}{2|v|}\left(\varepsilon_{z} \varepsilon_{z} \varepsilon_{x x}+\varepsilon_{x} \varepsilon_{x} \varepsilon_{z z}-\varepsilon_{z} \varepsilon_{x} \varepsilon_{x z}-\varepsilon_{x} \varepsilon_{z} \varepsilon_{z x}\right) \text {. }
$$

In experiments $h_{z}$ is related to the measurement: $J^{x}=e^{3} \gamma^{x z y} B^{z} E^{y}$. In the same manner $h_{x}$ and $h_{y}$ are related to the measurements: $J^{y}=e^{3} \gamma^{y x z} B^{x} E^{z}$ and $J^{z}=e^{3} \gamma^{z y x} B^{y} E^{x}$ where

$$
\gamma^{y x z}=\int \frac{\mathrm{d} S}{(2 \pi)^{3}} h_{x} \tau^{2}
$$

and

$$
\gamma^{z y x}=\int \frac{\mathrm{d} S}{(2 \pi)^{3}} h_{y} \tau^{2} .
$$

By summing three experimental results with different configurations we obtain

$$
\gamma^{x z y}+\gamma^{y x z}+\gamma^{z y x}=\int \frac{\mathrm{d} S}{(2 \pi)^{3}} H l^{2},
$$

where $l^{2}=|v|^{2} \tau^{2}$. This relation holds for any shape of the Fermi surface.

In the case of cubic symmetry (24) reduces to the Tsuji formula

$$
\gamma^{x z y}=\gamma^{y x z}=\gamma^{z y x}=\int \frac{\mathrm{d} S}{(2 \pi)^{3}} \frac{H}{3} l^{2}
$$

Now we are at the position from which we can guess why Haldane expected (14). In the case of cubic symmetry the integrand in $(25)$ is the product of the scalar $H$ and the square of the mean free path $l^{2}$. Haldane introduced a generalized expression by replacing the scalar with a tensor keeping $l^{2}$ in the integrand. However, in the case of general symmetry, $l^{2}$ cannot be factored out. We can only factor out $\tau^{2}$.

\section{Ong formula in 2D}

Although Haldane [3] discusses the relation between 2D and 3D formulae, we shall discuss the $2 \mathrm{D}$ case as a separate issue.

For simplicity we put $\boldsymbol{B}=(0,0, B)$ and set the $2 \mathrm{D}$ system in $x y$-plane. The 2D version of (1) on the Fermi line is given as

$$
\sigma^{x y}=e^{3} \int \frac{\mathrm{d} k_{t}}{(2 \pi)^{2}} l^{x}\left[(\boldsymbol{B} \times \boldsymbol{n}) \cdot \frac{\partial}{\partial \boldsymbol{k}}\right]^{z} l^{y},
$$

where $\mathrm{d} k_{t}$ is the length along the Fermi line. Since $\boldsymbol{B} \times \boldsymbol{n}=B \boldsymbol{t}$ and $\boldsymbol{t} \cdot(\partial / \partial \boldsymbol{k})=\partial / \partial k_{t}$, $(\boldsymbol{B} \times \boldsymbol{n}) \cdot(\partial / \partial \boldsymbol{k})=B \partial / \partial k_{t}$ where $\boldsymbol{t}$ is the unit tangent vector along the Fermi line, Eq. (26) is written as

$$
\sigma^{x y}=\frac{e^{3} B}{(2 \pi)^{2}} \int l^{x} \mathrm{~d} l^{y}
$$


where $\mathrm{d} l^{y}=\left(\partial l^{y} / \partial k_{t}\right) \mathrm{d} k_{t}$. After the anti-symmetrization we obtain the Ong formula

$$
\hat{\sigma}^{x y}=\frac{e^{3} B}{(2 \pi)^{2}} \int \frac{1}{2}\left[l^{x} \mathrm{~d} l^{y}-l^{y} \mathrm{~d} l^{x}\right]=\frac{e^{3} B}{(2 \pi)^{2}} \int \frac{1}{2}[l \times \mathrm{d} l]^{z},
$$

where $\hat{\sigma}^{x y} \equiv\left(\sigma_{x y}-\sigma_{y x}\right) / 2$. Moreover, Ong [4] discussed the "Stokes" area in $\boldsymbol{l}$-space.

\section{Summary}

In this paper we have discussed the geometrical formulae for the Hall conductivity.

In $2 \mathrm{D}$ the Ong formula is expressed by the area in $l$-space. In 3D the Tsuji formula is expressed by the curvature of the Fermi surface in $\boldsymbol{k}$-space.

\section{A Curvature in differential forms}

In this appendix we review the minimum fundamentals of a smooth surface $\Sigma$ in 3D Euclidean space. (See, for example, §4.5 of Ref. [8].)

Let us choose a point $\boldsymbol{x}$ on the surface $\Sigma$ and consider the vector $\boldsymbol{n}$ normal to $\Sigma$ at $\boldsymbol{x}$. Then we move to another point $\boldsymbol{x}^{\prime}$ on $\Sigma$ and consider the normal vector $\boldsymbol{n}^{\prime}$ there. Both $\boldsymbol{n}$ and $\boldsymbol{n}^{\prime}$ are unit vectors. We assume that the movement is infinitesimally small so that both $\mathrm{d} \boldsymbol{x} \equiv \boldsymbol{x}^{\prime}-\boldsymbol{x}$ and $\mathrm{d} \boldsymbol{n} \equiv \boldsymbol{n}^{\prime}-\boldsymbol{n}$ are in the tangent plane at the point $\boldsymbol{x}$. The normalization $\boldsymbol{n} \cdot \boldsymbol{n}=1$ leads to $\mathrm{d} \boldsymbol{n} \cdot \boldsymbol{n}=0$.

The vectors in the tangent plane are expanded as $\mathrm{d} \boldsymbol{x}=\sigma_{1} \boldsymbol{e}_{1}+\sigma_{2} \boldsymbol{e}_{2}$ and $\mathrm{d} \boldsymbol{n}=$ $\omega_{1} \boldsymbol{e}_{1}+\omega_{2} \boldsymbol{e}_{2}$ where $\boldsymbol{e}_{1}$ and $\boldsymbol{e}_{2}$ are the basis vectors of the tangent plane. Here $\sigma_{1}, \sigma_{2}$, $\omega_{1}$ and $\omega_{2}$ are 1-forms. The 2-form $\sigma_{1} \sigma_{2}$ represents the element of area of $\Sigma$. The 2 -form $\omega_{1} \omega_{2}$ represents the element of area of the unit sphere. The Gaussian curvature $K$ is introduced as the magnification factor between two areas: $\omega_{1} \omega_{2}=K \sigma_{1} \sigma_{2}$.

Two sets of 1-forms are related by a symmetric matrix $(c=b)$ as

$$
\left(\begin{array}{l}
\omega_{1} \\
\omega_{2}
\end{array}\right)=\left(\begin{array}{ll}
a & b \\
c & d
\end{array}\right)\left(\begin{array}{l}
\sigma_{1} \\
\sigma_{2}
\end{array}\right) .
$$

The determinant of this matrix is the Gaussian curvature: $K=a d-b c$. The trace is related to the mean curvature $H: 2 H=a+d$. If this $2 \times 2$ matrix is diagonalized as

$$
\left(\begin{array}{cc}
\kappa_{1} & 0 \\
0 & \kappa_{2}
\end{array}\right)
$$

$K=\kappa_{1} \kappa_{2}$ and $2 H=\kappa_{1}+\kappa_{2}$. Here the eigenvalues, $\kappa_{1}$ and $\kappa_{2}$, are principal curvatures.

\section{B Components of $2 \times 2$ representation}

In this appendix we calculate the components of the $2 \times 2$ matrix in the Appendix A explicitly. (See, for example, $\S 8.2$ of Ref. [8].)

We give the point on the surface $\Sigma$ as $\boldsymbol{x}=\left(x^{1}, x^{2}, u\right)$ with $u=u\left(x^{1}, x^{2}\right)$. Accordingly $\mathrm{d} \boldsymbol{x}=\left(\mathrm{d} x^{1}, \mathrm{~d} x^{2}, \mathrm{~d} u\right)$ where $\mathrm{d} u=p_{1} \mathrm{~d} x^{1}+p_{2} \mathrm{~d} x^{2}$ with $p_{i} \equiv \partial u / \partial x^{i}(i=1,2)$. Introducing 
the vectors $\boldsymbol{t}_{1}=\left(1,0, p_{1}\right)$ and $\boldsymbol{t}_{2}=\left(0,1, p_{2}\right)$ the small tangent vector is written as $\mathrm{d} \boldsymbol{x}=$ $\boldsymbol{t}_{1} \mathrm{~d} x^{1}+\boldsymbol{t}_{2} \mathrm{~d} x^{2}$. The unit normal vector is given by $\boldsymbol{n}=\boldsymbol{w} /|\boldsymbol{w}|$ with $\boldsymbol{w}=\left(-p_{1},-p_{2}, 1\right)$. It is apparent that $\boldsymbol{w} \cdot \boldsymbol{t}_{1}=0$ and $\boldsymbol{w} \cdot \boldsymbol{t}_{2}=0$.

Let us consider the map $\hat{A}: \mathrm{d} \boldsymbol{x} \rightarrow \mathrm{d} \boldsymbol{n}$ and introduce the $2 \times 2$ representation by

$$
\left(\mathrm{d} \boldsymbol{n} \cdot \boldsymbol{t}_{i}\right)=\sum_{j=1}^{2} a_{i j}\left(\mathrm{~d} \boldsymbol{x} \cdot \boldsymbol{t}_{j}\right)
$$

where the inner product is defined as

$$
\boldsymbol{x} \cdot \boldsymbol{t}=(x, y, z) \cdot\left(t^{x}, t^{y}, t^{z}\right)^{\mathrm{t}}=(x, y, z) \cdot\left(\begin{array}{l}
t^{x} \\
t^{y} \\
t^{z}
\end{array}\right)=x t^{x}+y t^{y}+z t^{z} .
$$

Since $\mathrm{d} \boldsymbol{n} \cdot \boldsymbol{t}_{i}=-(1 /|\boldsymbol{w}|) \sum_{j} r_{i j} \mathrm{~d} x^{j}$ and $\mathrm{d} \boldsymbol{x} \cdot \boldsymbol{t}_{j}=\sum_{k}\left(\delta_{j k}+p_{j} p_{k}\right) \mathrm{d} x^{k}$ with $r_{i j}=$ $\partial^{2} u / \partial x^{i} \partial x^{j}, a_{i j}$ satisfies

$$
\sum_{j=1}^{2} a_{i j}\left(\delta_{j k}+p_{j} p_{k}\right)=-\frac{1}{|\boldsymbol{w}|} r_{i k}
$$

In terms of Monge's notation $\left(p=\partial u / \partial x, q=\partial u / \partial y, r=\partial^{2} u / \partial x^{2}, s=\partial^{2} u / \partial x \partial y, t=\right.$ $\partial^{2} u / \partial y^{2}$ with $x^{1}=x$ and $x^{2}=y$ ) Eq. (33) is written as

$$
\hat{A}\left(\begin{array}{cc}
1+p^{2} & p q \\
p q & 1+q^{2}
\end{array}\right)=-\frac{1}{|\boldsymbol{w}|}\left(\begin{array}{ll}
r & s \\
s & t
\end{array}\right) .
$$

Thus

$$
\hat{A}=\frac{1}{|\boldsymbol{w}|^{3}}\left(\begin{array}{cc}
p q s-\left(1+q^{2}\right) r & p q r-\left(1+p^{2}\right) s \\
p q t-\left(1+q^{2}\right) s & p q s-\left(1+p^{2}\right) t
\end{array}\right) .
$$

The trace is readily obtained as

$$
2 H=\operatorname{trace}(\hat{A})=\frac{1}{|\boldsymbol{w}|^{3}}\left[2 p q s-\left(1+p^{2}\right) t-\left(1+q^{2}\right) r\right] .
$$

After some calculations the determinant is obtained as

$$
G=\operatorname{det}(\hat{A})=\frac{1}{|\boldsymbol{w}|^{4}}\left[r t-s^{2}\right] .
$$

The components $a_{i j}$ are also obtained by the derivative of the unit normal vector $\boldsymbol{n}=\left(n^{x}, n^{y}, n^{z}\right)$ where $\boldsymbol{n}=\boldsymbol{w} / w$ with $\boldsymbol{w}=(-p,-q, 1)$ and $w^{2} \equiv p^{2}+q^{2}+1$. Here we put $p_{x} \equiv r, q_{y} \equiv t$ and $s \equiv p_{y}=q_{x}$ for the convenience of the calculation. If we set the view point at $(0,0, \infty)$, the identification, $a_{11}=\partial n^{x} / \partial x, a_{21}=\partial n^{x} / \partial y, a_{12}=\partial n^{y} / \partial x$ and $a_{22}=\partial n^{y} / \partial y$, is naturally understood. The results are

$$
a_{11}=\frac{\partial n^{x}}{\partial x}=-\frac{1}{w} p_{x}+\frac{p}{w^{3}}\left(p p_{x}+q q_{x}\right)=\frac{1}{w^{3}}\left[p q s-\left(1+q^{2}\right) r\right],
$$




$$
\begin{aligned}
& a_{21}=\frac{\partial n^{x}}{\partial y}=-\frac{1}{w} p_{y}+\frac{p}{w^{3}}\left(p p_{y}+q q_{y}\right)=\frac{1}{w^{3}}\left[p q t-\left(1+q^{2}\right) s\right], \\
& a_{12}=\frac{\partial n^{y}}{\partial x}=-\frac{1}{w} q_{x}+\frac{q}{w^{3}}\left(p p_{x}+q q_{x}\right)=\frac{1}{w^{3}}\left[p q r-\left(1+p^{2}\right) s\right], \\
& a_{22}=\frac{\partial n^{y}}{\partial y}=-\frac{1}{w} q_{y}+\frac{q}{w^{3}}\left(p p_{y}+q q_{y}\right)=\frac{1}{w^{3}}\left[p q s-\left(1+p^{2}\right) t\right] .
\end{aligned}
$$

Here we should take care that $\mathrm{d} \boldsymbol{n}=\mathrm{d} \boldsymbol{x} \hat{A}$.

\section{C $3 \times 3$ representation}

Here we move from $\boldsymbol{x}$-space to $\boldsymbol{k}$-space. In the Appendix B we have assumed that the $z$-component is given by the function $u(x, y)$ explicitly. In the following we assume that the point $\boldsymbol{k}=\left(k^{x}, k^{y}, k^{z}\right)$ on the Fermi surface is given by $\varepsilon(\boldsymbol{k})=0$ implicitly.

The $2 \times 2$ matrix introduced in the Appendix B is written as

$$
\left(\begin{array}{ll}
\kappa^{x x} & \kappa^{y x} \\
\kappa^{x y} & \kappa^{y y}
\end{array}\right)
$$

which is a part of the $3 \times 3$ matrix

$$
\left(\begin{array}{lll}
\kappa^{x x} & \kappa^{y x} & \kappa^{z x} \\
\kappa^{x y} & \kappa^{y y} & \kappa^{z y} \\
\kappa^{x z} & \kappa^{y z} & \kappa^{z z}
\end{array}\right),
$$

where

$$
\kappa^{a b} \equiv \frac{\partial n^{a}}{\partial k^{b}}
$$

with $a, b=x, y, z$.

If the $2 \times 2$ matrix is diagonalized as

$$
\left(\begin{array}{cc}
\kappa_{1} & 0 \\
0 & \kappa_{2}
\end{array}\right)
$$

then the $3 \times 3$ matrix is diagonalized as

$$
\left(\begin{array}{ccc}
\kappa_{1} & 0 & 0 \\
0 & \kappa_{2} & 0 \\
0 & 0 & 0
\end{array}\right),
$$

since both $\mathrm{d} \boldsymbol{n}$ and $\mathrm{d} \boldsymbol{x}$ are in the tangent plane so that the normal vector becomes the eigenvector of the $3 \times 3$ matrix with zero eigenvalue. Consequently the trace of the $3 \times 3$ matrix is equal to the trace of $2 \times 2$ matrix.

The components $\kappa^{a b}$ are expressed in terms of the derivative of the quasi-particle energy $\varepsilon$. For example,

$$
\kappa^{z z}=\frac{\partial n^{z}}{\partial k^{z}}=\frac{\partial}{\partial k^{z}} \frac{\varepsilon_{z}}{\left(\varepsilon_{x}^{2}+\varepsilon_{y}^{2}+\varepsilon_{z}^{2}\right)^{1 / 2}}=\frac{\varepsilon_{z z}}{|\boldsymbol{v}|}-\frac{\varepsilon_{z}}{|\boldsymbol{v}|^{3}}\left(\varepsilon_{x} \varepsilon_{x z}+\varepsilon_{y} \varepsilon_{y z}+\varepsilon_{z} \varepsilon_{z z}\right),
$$


where $|v|^{2}=\varepsilon_{x}^{2}+\varepsilon_{y}^{2}+\varepsilon_{z}^{2}$. In Eq. (47) the term $\varepsilon_{z} \varepsilon_{z} \varepsilon_{z z}$, which is not expected for the off-diagonal conductivity, disappers by the subtraction so that we obtain

$$
\kappa^{z z}=\frac{1}{|\boldsymbol{v}|^{3}}\left[\left(\varepsilon_{x} \varepsilon_{x}+\varepsilon_{y} \varepsilon_{y}\right) \varepsilon_{z z}-\varepsilon_{z}\left(\varepsilon_{x} \varepsilon_{x z}+\varepsilon_{y} \varepsilon_{y z}\right)\right] .
$$

In the same manner we also obtain

$$
\kappa^{x x}=\frac{1}{|v|^{3}}\left[\left(\varepsilon_{y} \varepsilon_{y}+\varepsilon_{z} \varepsilon_{z}\right) \varepsilon_{x x}-\varepsilon_{x}\left(\varepsilon_{y} \varepsilon_{y x}+\varepsilon_{z} \varepsilon_{z x}\right)\right],
$$

and

$$
\kappa^{y y}=\frac{1}{|v|^{3}}\left[\left(\varepsilon_{x} \varepsilon_{x}+\varepsilon_{z} \varepsilon_{z}\right) \varepsilon_{y y}-\varepsilon_{y}\left(\varepsilon_{x} \varepsilon_{x y}+\varepsilon_{z} \varepsilon_{z y}\right)\right]
$$

\section{References}

[1] M. Tsuji, J. Phys. Soc. Jpn. 13, 979 (1958).

[2] C. M. Hurd, The Hall Effect in Metals and Alloys (Plenum Press, New York, 1972).

[3] F. D. M. Haldane, arXiv:cond-mat/0504227v2.

[4] N. P. Ong, Phys. Rev. B 43, 193 (1991).

[5] J. M. Ziman, Electrons and Phonons (Clarendon Press, Oxford, 1960).

[6] G. Kotliar, A. Sengupta and C. M. Varma, Phys. Rev. B 53, 3573 (1996).

[7] H. Kohno and K. Yamada, Prog. Theor. Phys. 80, 623 (1988).

[8] H. Flanders, Differential Forms (Academic Press, New York, 1963). 